Letter to the Editor

\title{
Response to the letter "Handgrip strength may not accurately reflect the overall nutritional status of patients"
}

\section{Dear Editors,}

We read with great interest the very punctual comments regarding our recently published article entitled "Handgrip strength is an independent predictor of all-cause mortality in maintenance dialysis patients" [1] in the letter "Handgrip strength may not accurately reflect the overall nutritional status of patients" by Shi Bo and Chen Junqiang.

In fact, malnutrition is an important problem in the course of chronic diseases progression. However, the causes and mechanisms involved in malnutrition in chronic kidney disease (CKD) are not the same as cancer. Particularly, in chronic kidney disease, malnutrition is characterized by loss of muscle mass, which is often associated with diminished functional capacity [2] and quality of life [3], morbidity, and all-cause mortality [3-5]. The terminology for malnutrition characteristic of CKD is "protein energy wasting" (PEW) [2]. Because of the significant role on mortality prediction, we consider strictly important to detect even the early stages of PEW. It is not of our knowledge other cheap and feasible assessments that can predict the very early stages of malnutrition such as handgrip strength. We would not diminish the importance of an assessment that can detect mortality risk earlier than low muscle mass in dialysis patients (evaluated in our study by middle-arm muscle circumference, which was not significantly different between survival and non-survival patients). This would permit the care team to provide early intervention, and thus, try to improve the poor prognosis.

As muscle mass loss is the main characteristic of PEW [2], and as muscle strength loss precedes muscle mass loss [6], the assessment of muscle strength is relevant in dialysis patients. We have not cited anytime in our paper that muscle strength may reflect the overall nutritional status of dialysis patients, although associations between handgrip and malnutrition inflammation score, which is a score specifically developed for CKD patients that assess the overall nutritional status, were verified in CKD [7] and dialysis patients [8].

To the best of our knowledge, there is no study evaluating if muscle strength and muscle function changes reflect only the early stages of PEW, not the overall nutritional status of patients. We hypothesize that muscle strength progressively decrease with

DOI of original article: http://dx.doi.org/10.1016/j.clnu.2016.11.016. concurrent muscle mass loss. However, studies with longitudinal assessments evaluating the influence of muscle strength changes on mortality risk are necessary.

We agree that patients who cannot perform handgrip strength measurement should be excluded of the study. We know it would be an important bias. In our cohort, none of the included patients presented any of those conditions (shoulder injury, arm or hand injury, chest and abdominal surgery, ipsilateral breast cancer radical operation, Parkinson's disease, brachial plexus injury, cervical spondylotic radiculopathy with numbness, and thoracic outlet syndrome).

We also do not know any study that shows an association of nutritional status with variation in handgrip strength in CKD and dialysis patients and unfortunately, there are no references in your letter. Some studies evaluate a single measurement of handgrip strength as predictor of renal outcome [9], inflammation [10] and mortality [10-12].

We agree the term "decreased handgrip strength" refers to an evolution of handgrip strength. Perhaps none of the authors either the peer reviewers noted this small contradiction, since this term is cited only once in our paper. We can't agree that "decreased handgrip strength" is used for nutritional assessment, and "low handgrip strength" is used for nutrition screening. We can use "decreased handgrip strength" to compare the change on handgrip strength between nutritional assessments, while "low handgrip strength" also might be used in a single nutritional assessment, as well for nutrition screening.

We appreciate the opportunity to answer it, mainly because we believe it is necessary to discuss and clarify the issues raised in their letter. However, it's a cause for concern that in massive publication days, the preciosity is often highly valued, leading to expand minor details and generating delay in demands and lack of focus on really strategic information.

\section{Conflict of interest statement}

None.

\section{References}

[1] Vogt BP, Borges MCC, Goés CR, Caramori JCT. Handgrip strength is an independent predictor of all-cause mortality in maintenance dialysis patients. Clin Nutr 2016;35:1429-33. 
[2] Fouque D, Kalantar-Zadeh K, Kopple J, Cano N, Chauveau P, Cuppari L, et al. A proposed nomenclature and diagnostic criteria for protein-energy wasting in acute and chronic kidney disease. Kidney Int 2008;73:391-8.

[3] Noori N, Kopple JD, Kovesdy CP, Feroze U, Sim JJ, Murali SB, et al. Mid-arm muscle circumference and quality of life and survival in maintenance hemodialysis patients. Clin J Am Soc Nephrol 2010;5:2258-68.

[4] Antunes AA, Vannini FD, Silveira LVA, Martin LC, Barretti P, Caramori JC. Influence of protein intake and muscle mass on survival in chronic dialysis patients. Ren Fail 2010;32:1055-9.

[5] Araujo IC, Kamimura MA, Draibe SA, Canziani MEF, Manfredi SR, Avesani CM, et al. Nutritional parameters and mortality in incident hemodialysis patients. J Ren Nutr 2006;16:27-35.

[6] Carrero JJ, Johansen KL, Lindholm B, Stenvinkel P, Cuppari L, Avesani CM. Screening for muscle wasting and dysfunction in patients with chronic kidney disease. Kidney Int 2016;90:53-66.

[7] Amparo FC, Cordeiro AC, Carrero J, Cuppari L, Lindholm B, Amodeo C, et al Malnutrition-inflammation score is associated with handgrip strength in nondialysis-dependent chronic kidney disease patients. J Ren Nutr 2013;23: $283-7$.

[8] Silva LF, Matos CM, Lopes GB, Martins MTS, Martins MS, Arias LU, et al. Handgrip strength as a simple indicator of possible malnutrition and inflammation in men and women on maintenance hemodialysis. J Ren Nutr 2011;21:235-45.

[9] Chang Y-T, Wu H-L, Guo H-R, Cheng Y-Y, Tseng C-C, Wang M-C, et al. Handgrip strength is an independent predictor of renal outcomes in patients with chronic kidney diseases. Nephrol Dial Transpl 2011;26:3588-95.
[10] Wang AY-M, Sea MM-M, Ho ZS-Y, Lui S-F, Li PK-T, Woo J. Evaluation of handgrip strength as a nutritional marker and prognostic indicator in peritoneal dialysis patients. Am J Clin Nutr 2005;81:79-86.

[11] Isoyama N, Qureshi AR, Avesani CM, Lindholm B, Barany P, Heimbürger O, et al. Comparative associations of muscle mass and muscle strength with mortality in dialysis patients. Clin J Am Soc Nephrol 2014;9:1720-8.

[12] Matos CM, Silva LF, Santana LD, Santos LS, Protasio BM, Rocha MT, et al. Handgrip strength at baseline and mortality risk in a cohort of women and men on hemodialysis: a 4-year study. J Ren Nutr 2014;24(3):157-62.

Barbara Perez Vogt, Mariana Clementoni Costa Borges, Cassiana Regina de Góes, Jacqueline Costa Teixeira Caramori"

Department of Clinical Medicine, Faculdade de Medicina de Botucatu, UNESP Univ Estadual Paulista, Brazil

* Corresponding author. E-mail address: jteixeir@fmb.unesp.br (J.C.T. Caramori).

14 November 2016 